\title{
The isolation and characterization of Duck astrovirus type- 1remerging in China
}

\author{
Feng Wei ${ }^{1}$, Yueming Wang ${ }^{1}$, shuai Zhang ${ }^{1}$, Jing Yang $^{2}$, Xiang Diao ${ }^{3}$, and YI TANG ${ }^{1}$ \\ ${ }^{1}$ Affiliation not available \\ ${ }^{2}$ AgriculturalUniversity of Shan Dong province \\ ${ }^{3}$ Agricultural University of Shan Dong province
}

October 30, 2020

\begin{abstract}
Since the first report from Cherry Valley ducks on a commercial duck farm in China (2008), duck astrovirus type 1(DAstV-1) -associated duck viral hepatitis (DVH) have been detected in several commercial duck flocks. In the literature, no outbreak of DAstV-1 have been report in China since 2012. Here, the isolation, cultivation and characterization of DAstV-1 isolate are described. One DAstV-1 strain, designated as DAstV-SDZZ, was isolated from a diseased duckling. The isolated astrovirus grew well in the LMH cell line. To determine the entire genomic of the DAstV-SDZZ isolate, next-generation sequencing (NGS) technique was conducted on Illumina HiSeq platform. Complete genome sequence analysis revealed that DAstV-SDZZ isolate was 91.6\%-98.7\% homology with others DAstV-1 deposited in Genbank. Similar clinical symptoms were successful reproduced by experimental infection study using the DAstV-SDZZ isolate. DAstV-SDZZ is the first DAstV-1 strain whose experimental infection study has been conducted. The present works are likely to provide new insights into the pathogenicity and evolution of DAstV-1 in ducks.
\end{abstract}

The isolation and characterization of Duck astrovirus type- 1remerging in China

Feng Wei ${ }^{* 1,2,3}$, Yueming Wang ${ }^{* 1,2,3}$, Jing Yang ${ }^{1,2,3}$, Shuai Zhang ${ }^{1,2,3}$, Youxiang Diao*1,2,3 , Yi Tang $^{* 1,2,3}$

College of Animal Science and Technology, Shandong Agricultural University,61 Daizong Street, Tai'an, Shandong Province, 271018, China

2. Shandong Provinecial Key Laboratory of Animal Biotechnology and Disease Control and Prevention, Tai'an, Shandong, 271018, China

3.Shandong Provincial Engineering Technology Research Center of Animal Disease Control and Prevention, Tai'an, Shandong, 271018, China

Email: Feng Wei: 2947732597@qq.com

Email: Yueming Wang: 185984839@qq.com

Email: Jing Yang: yj9210@163.com

Email: Shuai Zhang: zxf4716@163.com

Correspondence:

Email: Youxiang Diao: yxdiao@163.com

Email: Yi Tang: tyck288@163.com 


\begin{abstract}
Since the first report from Cherry Valley ducks on a commercial duck farm in China (2008), duck astrovirus type $1(\mathrm{DAstV}-1)$-associated duck viral hepatitis (DVH) have been detected in several commercial duck flocks. In the literature, no outbreak of DAstV-1 have been report in China since 2012. Here, the isolation, cultivation and characterization of DAstV-1 isolate are described. One DAstV-1 strain, designated as DAstVSDZZ, was isolated from a diseased duckling. The isolated astrovirus grew well in the LMH cell line. To determine the entire genomic of the DAstV-SDZZ isolate, next-generation sequencing (NGS) technique was conducted on Illumina HiSeq platform. Complete genome sequence analysis revealed that DAstV-SDZZ isolate was 91.6\%-98.7\% homology with others DAstV-1 deposited in Genbank. Similar clinical symptoms were successful reproduced by experimental infection study using the DAstV-SDZZ isolate. DAstV-SDZZ is the first DAstV-1 strain whose experimental infection study has been conducted. The present works are likely to provide new insights into the pathogenicity and evolution of DAstV-1 in ducks.
\end{abstract}

Keywords: duck astrovirus type 1; next-generation sequencing; complete gene sequence; phylogenetic analysis; pathogenicity

\title{
Introduction
}

Astroviruses are non-enveloped, positive-sense and single-stranded RNA viruses with a diameter of approximately 28-30 nm (Baxendale and Mebatsion, 2004; Fauquet et al., 2005). Their single-stranded RNA genome ranges in size from 6.8 to $7.9 \mathrm{~kb}$ and consisting of three open reading frames (ORFs) ; ORF 1a, ORF 1b and ORF 2 (Fu et al., 2008; De Benedictis et al., 2011). ORF1a codes for non-structural (NS) polyprotein, which include a serine protease, several transmembrane (TM) helical motifs and a nuclear localization signal (NLS) (Jiang et al., 1993; Lee and Kurtz, 1994). ORF 1b encodes the RNA-dependent RNA polymerase (Willcocks et al., 1994), while ORF2 codes for the viral capsid structural protein that is required for virion formation (Arias and Dubois, 2017).

In poultry, astroviruses have been reported to infect several avian species, including chickens (Kang et al., 2018), turkeys (Pantin-Jackwood et al., 2006), gooses (Q. Zhang et al., 2018; Yang et al., 2018) and ducks (Chen et al., 2012; Wei et al., 2020). Astroviruses disease in most species are causative agents of enteric diseases. In ducks, unlike other avian species, astroviruses have been associated with a fatal hepatitis, historically known to be caused by two types of duck hepatitis virus (DHV-2 and DHV-3) which were considered picornaviruses (Asplin, 1965; Haider and Calnek, 1979). More recently, DHV-2 and DHV-3 has been identified as astrovirus based on a $391 \mathrm{nt}$ ORF $1 \mathrm{~b}$ region (Todd et al., 2009). Besides the two types of formal classification duck astrovirus (DAstV), there are a number of unassigned duck astrovirus waiting to be classified, such as duck DHV-3-like astroviruses (Liu, Wang, Shi et al., 2014), duck astrovirus CPH (DAstV CPH) (Liao et al., 2015), duck astrovirus YP-like and astroviruses detected from wild birds (Chu et al., 2012; Liu, Wang and Zhang, 2014).

In China, duck viral hepatitis is mainly caused by duck hepatitis A virus (DHAV) (Wen et al., 2018). No outbreaks of DAstV-1 have been published reports in China since 2012 (Chen et al., 2012). Therefore, little is known regarding their molecular biology and viral genetic diversity. In addition, due to the lack of effective methods for cultivating DAstV-1, the pathogenicity of the DAstV-1 in ducklings remains unclear. The present paper describes the molecular characterization of this virus and phylogenetic analysis to other astroviruses. The reproduction experiment was conducted to investigate the pathogenicity of the virus.

\section{Materials and methods}

\section{Ethics statement}

All animal infection experiments were conducted in accordance with the "Guidelines for Experimental Animals" of the Ministry of Science and Technology (Beijing, China) and were conducted under the supervision of the Animal Protection and Utilization Committee of Shandong Agricultural University.

\section{Clinical samples}


The disease started in 1 to 3 -week-old ducks on rearing fields with mortality rates ranging from 10 to $30 \%$. Their clinical signs included lethargy, loss of appetite and weight loss. Postmortem examination revealed a steatosis and haemorrhagic lesions of the liver, as well as hemorrhage and swellings in kidneys. From May to September 2020, a total of one hundred and seventy liver samples were taken from the diseased ducks from different commercial duck farms located at four provinces of China (Figure1). The tissues from different commercial duck farms were homogenized in phosphate buffered saline (PBS) $(20 \%$, w/v) containing antibiotics (100 U/ml of Penicillin and $100 \mu \mathrm{g} / \mathrm{ml}$ of Streptomycin), and centrifuged at $12,000 \times \mathrm{g}$ at $4{ }^{\circ} \mathrm{C}$ for 15 minutes. The supernatant was filtered through a $0.22-\mu \mathrm{m}$ syringe-driven filter to remove large particles and stored at $-80^{\circ} \mathrm{C}$ until use. The existence of other avian pathogenic viruses (including AIV, TMUV, DHAV-1, DHAV-3, FAV, DPV, GPV, GoAstV and DucV) were ruled out by reverse transcription PCR (RT-PCR) or PCR.

\section{RNA extraction and RT-PCR}

Total RNA was extracted from liver filtrate by MiniBEST Universal RNA Extraction Kit (TaKaRa,Dalian, China), following the manufacturer's instructions. The extracted RNA was carried out using One-step RT-PCR Kit (TaKaRa, Dalian, China) with specific primers (DAstV-F 5'CTTGGACTGTGGAAGCATATACC-3', DAstV-R 5'-GTTGAAAACTGCCCTGAAGG-3') to amplify a partial sequence of approximately 763 bp of a DAstV-1, and PCR amplicons were sequenced for confirmation.

\section{Virus isolation and sequencing}

For virus isolation a selection of liver sample (ZaoZhuang County) from Shandong province that was tested positive for DAstV-1 by RT-PCR was prepared. $0.5 \mathrm{ml}$ of the filtered suspension was inoculated into LMH cells (ATCC CRL-2013), a chicken hepatocellular carcinoma cell line. The cultures incubated at 37with 5\% $\mathrm{CO}_{2}$ and examined for a cytopathic effect (CPE) daily. The cells and supernatant within 4-7 days post inoculation (PI) were snap-frozen for further passages.

\section{Next-generation sequencing}

The RNA extracted from the DAstV-SDZZ sample was subjected to complete genome sequencing by nextgeneration sequencing (NGS) with the Hiseq platform following the manufacturer's instructions. The NGS was performed at the Shanghai Personal Biotechnology Co., Ltd, China. The details of the NGS library preparation are the same as we described earlier (Wei et al., 2020). Specific primers were developed to verify the genomic sequence of the isolate as described previously (R. Zhang et al., 2019).

\section{Phylogenetic analysis}

Phylogenetic analysis based on the nucleotide and deduced amino acid sequences of DAstV-SDZZ isolate, was performed using ClustalW in the Megalin module within the Lasergene 7.0 software (DNASTAR Inc., Madison WI, USA) to determine the genetic relationships among DAstV strains. Phylogenetic trees of the three ORFs were done by the neighbor-joining method implemented in MEGA version 7.0 program, with bootstrap values calculated from 1000 replicates.

\section{Experimental infection study}

The aim of the study was conducted to determine the pathogenicity of the isolate. The specific pathogen free (SPF) duck embryos were obtained from a hatchery (Harbin Veterinary Research Institute). Newly hatched SPF ducklings were moved in SPF negative pressured isolators. Twenty 1-day-old SPF ducklings were infected by subcutaneous injection with $0.2 \mathrm{ml}$ of the isolated virus (DAstV-SDZZ strain; $\left.5 \times 10^{4.8} \mathrm{TCID}_{50} / 0.1 \mathrm{ml}\right)$ as the experimental group, while the other ducklings $(\mathrm{n}=20)$ were inoculated with sterile phosphate buffered saline $(0.2 \mathrm{~mL})$ in the same manner as the control group. Clinical signs and mortality of ducklings were monitored daily. Survival birds were euthanized at 15 days after inoculation. Dead birds were necropsied immediately for histopathological observation and virus identification. All ducklings were bred and cared for in accordance with humane procedures. 


\section{Histological examination}

Portions fresh tissues (e.g., liver and kidney) from infected ducks were fixed with a10\% neutral buffered formalin, sectioned into $4-5 \mu \mathrm{m}$ thick with paraffin and stained with haematoxylin and eosin using standard methods. Tissues samples (e.g., liver and kidney) from the diseased or experimental ducklings were frozen for virus isolation and identification.

\section{Results}

\section{Viral nucleic acids detection in clinical samples}

Conventional infections with avian pathogenic viruses were ruled out. In all liver samples examined. DNA fragments of approximately 763 bp were amplified from $95.8 \%(163 / 170)$ of liver samples by a pair of specific primers of DAstV-1. The same product was obtained in the positive control. In contrast, no amplicon was observed in negative control.

\section{Virus isolation}

The liver sample (ZaoZhuang County) from Shandong province was selected for virus isolation. After five passages, an astrovirus designated as DAstV/SDZZ/Duck (DAStV-SDZZ) strain, was successfully isolated. Most inoculated cells showed CPE after inoculation within $72-96 \mathrm{hr}$ and tested positive for DAStV-1 by RT-PCR using the above primers.

\section{NGS analysis}

A total of 1,735,463 sequencing reads were generated by the HiSeq sequencer from extracted viral total RNA, and yielding $376 \mathrm{Mb}$ of fastq format sequenced data. After processed by quality control (QC) filters of the Hiseq platform, non-research target readings that were similar to mRNA or rRNA sequences were removed. The residual 231,327 clean reads were assembled by de novo assembly module of CLC Genomic Workbench software (version 10.0). Finally, one long contig that covered the full length of an astrovirus was generated by re-mapping the clean reads back to the astrovirus reference sequences. Whereas no other pathogen was discovered by analysing all assembled contigs.

\section{Genome sequence analysis}

As revealed from the sequencing results, the complete genome of DAstV-SDZZ comprised 7,757 nucleotides (nt) with a 34-nt poly(A) tail, and was submitted to the GenBank (accession number MN809622), making it the largest among astroviruses so far sequenced. The coding region of DAstV-SDZZ strain consisting of three overlapping ORFs of 3,723 nt (ORF1a), 1,551 nt (ORF1b) and 2,196 nt (ORF2), as well as a short 5' UTR of 22 nt and a 3' UTR of 252 nt. The three sequential ORFs encoded polypeptides of 1,240 (positions 23 to 3745 ), 516 (positions 3736 to 5286 ), and 731 (positions 5310 to 7505 ) amino acids, respectively. Furthermore, a ribosomal frameshift signal was observed in the overlap region between ORF1a and ORF1b of DAstV-SDZZ, consisting of the heptameric sequence AAAAAAC from nt 3736 to 3742.

\section{Phylogenetic analysis}

To investigate the evolutionary relationship of DAstV -SDZZ with other astroviruses for which corresponding sequences were available, phylogenetic trees were constructed based on nucleotide sequences of the ORF 1a, ORF $1 \mathrm{~b}$ and ORF 2 homologous genome (Figure 2). All the three trees demonstrated that the DAstV -SDZZ isolate located on the identical branch as the other DAstV-1. The DAstV-1 strain was closely related to some turkey astroviruses in ORF 1a and ORF 2 genes, whereas DAstV-1 formed another clade with CAstV and DAstV CPH in the ORF2 region. Comparison of the other DAstV-1 strain with the deduced amino acid sequences of DAstV-SDZZ isolate were performed. As shown in Table 1, the deduced amino acid sequences of the DAstV-SDZZ with other DAstV-1 strain shared identities of $94.4 \%$ to $99 \%$ in ORF1a, 96.9\% to $98.4 \%$ in ORF1b, and $95.5 \%$ to $99.9 \%$ in ORF2. The amino acid of capsid protein of the DAstV-SDZZ shared identities of $40.5 \%$ and $56-56.1 \%$ with TAstV-1 and GoAstV strains, respectively. These observations suggest that DAstV-SDZZ is an isolate of DAstV-1. 


\section{Pathogenicity of DAstV-SDZZ in ducklings}

Three of 20 ducklings inoculated with the virus died between 4 and 6 days post infection (dpi), with most infected ducklings exhibited signs of depression on the $2^{\text {nd }}$ day. As a result, a mortality rate of $15 \%$ was achieved (3/20). At necropsy, the liver showed petechial and ecchymosis hemorrhages (Figure 3A). Besides, enlarged kidneys with congestion were variable in dead ducklings (Figure 3B). Significant histologic changes in the kidneys were noted from the infected group, included necrosis and degeneration of renal epithelial cells, accompanied by infiltration of inflammatory cells and severe haemorrhage (Figure 3D). Besides, steatosis and infiltration of inflammatory cells were observed in the livers (Figure 3C). No histological microscopic lesions were seen in the control group.

\section{Discussion}

In naturally occurring outbreaks, DVH is mainly caused by DHAV and causes great economic losses (Niu et al., 2019). Several studies have reported the existence of DAstV-1 in commercial duck farms (Fu et al., 2009; Chen et al., 2012). Unfortunately, the pathogenicity of DAstV-1 among ducklings remains unclear due to the lack of efficient in vitro culture techniques. In the present study, we report the isolation and characterization of DAstV-1 strain isolated from ducklings in China, and reproduction experiment of the disease in ducklings with the inoculating virus prepared in LMH cells has been carried out.

The infection study with DAstV-SDZZ isolate were successful reproduced. Although DAstV-1 infection has been detected in several commercial duck flocks. Unfortunately, attempts to isolate the DAstV-1 virus were unsuccessful. To the best of our knowledge, DAstV-SDZZ is the first DAstV-1 strain whose experimental infection study has been conducted. In this study, the isolated astrovirus grew well in LMH cells, the CPE appeared after inoculation within 72-96 hr. Existing studies have shown that some astroviruses can be serially passaged only after being cleaved by trypsin to form mature virions (Bass and Qiu, 2000; Mendez et al., 2007). In this study, DAstV-SDZZ isolate grew well in LMH cells without the presence of trypsin, but it does not rule out that the presence of trypsin can increase the titer of the virus to a certain extent.

In a previous study,DAstV-1 infection caused a mortality rate of about $50 \%$ in large groups, and dead duckling displaying the typical haemorrhagic lesions in the liver (Fu et al., 2009). Obviously, the disease described in the present study was distinct from the reports in the literature in terms of signs, lesions, and mortality rate. From field cases and experimental studies, major lesions were demonstrated in the kidneys which are hemorrhage and swellings. This may be due to the mutation of some key sites of the virus has caused the change of pathogenicity and tissue tropism. At the same time, the virus has a stronger adaptability to the host so that it can spread widely.

Previous studies showed that the poly (A) tail of the positive single-stranded RNA virus genome plays a very important role in its translation, replication and transcription (Bergamini et al., 2000; Kean, 2003). In addition, it was shown in another study that the length of the poly(A) tail plays a direct role in viral RNA replication in infected cells since reducing the length of the poly(A) tail resulted in a reduction in RNA infectivity (Silvestri et al., 2006). An important finding of the current study is that the length of the poly(A) tail of DAstV-SDZZ isolate is the largest among duck astroviruses so far sequenced. All of these indicate that a similar relationship may exists between DAstV-1strain infectivity and the length of poly(A) tail, which promotes the widespread of the virus. Further studies are needed to clarify those prediction.

In summary, DAstV-1 infections are more common than previously known in China. Thus, a continued surveillance of DAstV-1 is required to understand the molecular epidemiology of the viruses circulating in China. However, the limitation of our study was that we did not provide scientific evidence for the molecular epidemiology and changes in virus transmission capacity of DAstV-1 in China. Nevertheless, this study provides scientific evidence of the DAstV-1 could be one of the pathogenic factors prevalent in duck flocks in China.

\section{Acknowledgement}


This work was supported by the National Key Research and Development Program of China (2018YFD0501506);

The Natural Science Foundation of Shandong Province (ZR2019YQ17);

The Higher Education Support Program of Youth Innovation and Technology of Shandong Province, China(2019KJF022);

The China Agriculture Research System (CARS-42-19);

Funds of Shandong "Double Top" Program (SYL2017YSTD11).

\section{Author details}

College of Animal Science and Technology, Shandong Agricultural University, 61 Daizong Street, Taian, Shandong Province, 271018, China. ${ }^{1}$

Shandong Provinecial Key Laboratory of Animal Biotechnology and Disease Control and Prevention, Taian, Shandong, 271018, China. ${ }^{2}$

Shandong Provincial Engineering Technology Research Center of Animal Disease Control and Prevention, Taian, Shandong, 271018, China. ${ }^{3}$

\section{Conflict of interest}

The authors declare that the research was conducted in the absence of any commercial or financial relationship that could be construed as a potential conflict of interest. Author Contributions Conceived and designed the experiments: YT YD FW. Performed the experiments: FW MW. Analyzed the data: FW. Contributed reagents/materials/analysis tools: SZ JY. Wrote the paper: FW.

\section{Data Availability Statement}

The data that support the findings of this study are available from the corresponding author, Youxiang Diao: yxdiao@163.com; Yi Tang: tyck288@163.com, upon reasonable request.

\section{Reference}

Arias, C.F., and R.M. Dubois, 2017: The astrovirus capsid: A review. Viruses DOI: 10.3390/v9010015.

Asplin, F.D., 1965: Duck hepatitis: vaccination against two serological types. Vet. Rec. DOI: 10.1136/vr.77.50.1529.

Bass, D.M., and S. Qiu, 2000: Proteolytic Processing of the Astrovirus Capsid. J. Virol. DOI: 10.1128/jvi.74.4.1810-1814.2000.

Baxendale, W., and T. Mebatsion, 2004: The isolation and characterisation of astroviruses from chickens. Avian Pathol. DOI: 10.1080/0307945042000220426.

Bergamini, G., T. Preiss, and M.W. Hentze, 2000: Picornavirus IRESes and the poly(A) tail jointly promote cap-independent translation in a mammalian cell-free system. RNA DOI: 10.1017/S1355838200001679.

Chen, L., Q. Xu, R. Zhang, J. Li, Z. Xie, Y. Wang, Y. Zhu, and S. Jiang, 2012: Complete Genome Sequence of a Duck Astrovirus Discovered in Eastern China. J. Virol. DOI: 10.1128/jvi.02637-12.

Chu, D.K.W., C.Y.H. Leung, H.K.K. Perera, E.M. Ng, M. Gilbert, P.H. Joyner, A. Grioni, G. Ades, Y. Guan, J.S.M. Peiris, and L.L.M. Poon, 2012: A Novel Group of Avian Astroviruses in Wild Aquatic Birds.J. Virol. DOI: $10.1128 /$ jvi.02105-12.

De Benedictis, P., S. Schultz-Cherry, A. Burnham, and G. Cattoli, 2011: Astrovirus infections in humans and animals - Molecular biology, genetic diversity, and interspecies transmissions. Infect. Genet. Evol. DOI: 10.1016/j.meegid.2011.07.024. 
Fauquet, C., M.A. Mayo, J. Maniloff, U. Desselberger, and L.A. Ball, 2005: Virus Taxonomy: Eighth Report of the International Committee on Taxonomy of Viruses.Virus Taxon. Eighth Rep. Int. Comm. Taxon. Viruses .

Fu, Y., M. Pan, X. Wang, Y. Xu, X. Xie, N.J. Knowles, H. Yang, and D. Zhang, 2009: Complete sequence of a duck astrovirus associated with fatal hepatitis in ducklings. J. Gen. Virol. DOI: 10.1099/vir.0.008599-0.

Fu, Y., M. Pan, X. Wang, Y. Xu, H. Yang, and D. Zhang, 2008: Molecular detection and typing of duck hepatitis A virus directly from clinical specimens. Vet. Microbiol. DOI: 10.1016/j.vetmic.2008.03.011.

Haider, S.A., and B.W. Calnek, 1979: In vitro isolation, propagation, and characterization of duck hepatitis virus type III. Avian Dis. DOI: 10.2307/1589748.

Jiang, B., S.S. Monroe, E. V. Koonin, S.E. Stine, and R.I. Glass, 1993: RNA sequence of astrovirus: Distinctive genomic organization and a putative retrovirus-like ribosomal frameshifting signal that directs the viral replicase synthesis. Proc. Natl. Acad. Sci. U. S. A. DOI: 10.1073/pnas.90.22.10539.

Kang, K. Il, E. Linnemann, A.H. Icard, V. Durairaj, E. Mundt, and H.S. Sellers, 2018: Chicken astrovirus as an aetiological agent of runting-stunting syndrome in broiler chickens. J. Gen. Virol. DOI: 10.1099/jgv.0.001025.

Kean, K.M., 2003: The role of mRNA 5'-noncoding and 3'-end sequences on 40S ribosomal subunit recruitment, and how RNA viruses successfully compete with cellular mRNAs to ensure their own protein synthesis.Biol. Cell DOI: 10.1016/S0248-4900(03)00030-3.

Lee, T.W., and J.B. Kurtz, 1994: Prevalence of human astrovirus serotypes in the Oxford region 1976-92, with evidence for two new serotypes. Epidemiol. Infect. DOI: 10.1017/S0950268800057551.

Liao, Q., N. Liu, X. Wang, F. Wang, and D. Zhang, 2015: Genetic characterization of a novel astrovirus in Pekin ducks. Infect. Genet. Evol. DOI: 10.1016/j.meegid.2015.02.025.

Liu, N., F. Wang, J. Shi, L. Zheng, X. Wang, and D. Zhang, 2014: Molecular characterization of a duck hepatitis virus 3-like astrovirus. Vet. Microbiol. DOI: 10.1016/j.vetmic.2014.01.026.

Liu, N., F. Wang, and D. Zhang, 2014: Complete sequence of a novel duck astrovirus. Arch. Virol. DOI: 10.1007/s00705-014-2141-0.

Mendez, E., G. Aguirre-Crespo, G. Zavala, and C.F. Arias, 2007: Association of the Astrovirus Structural Protein VP90 with Membranes Plays a Role in Virus Morphogenesis. J. Virol. DOI: 10.1128/jvi.00785-07.

Niu, Y., H. Ma, Y. Ding, Z. Li, Y. Sun, M. Li, and Y. Shi, 2019: The pathogenicity of duck hepatitis A virus types 1 and 3 on ducklings.Poult. Sci. DOI: 10.3382/ps/pez455.

Pantin-Jackwood, M.J., E. Spackman, and P.R. Woolcock, 2006: Molecular characterization and typing of chicken and turkey astroviruses circulating in the United States: Implications for diagnostics.Avian Dis. DOI: 10.1637/7512-020606R.1.

Silvestri, L.S., J.M. Parilla, B.J. Morasco, S.A. Ogram, and J.B. Flanegan, 2006: Relationship between poliovirus negative-strand RNA synthesis and the length of the 3' poly(A) tail. Virology DOI: 10.1016/j.virol.2005.10.019.

Todd, D., V.J. Smyth, N.W. Ball, B.M. Donnelly, M. Wylie, N.J. Knowles, and B.M. Adair, 2009: Identification of chicken enterovirus-like viruses, duck hepatitis virus type 2 and duck hepatitis virus type 3 as astroviruses. Avian Pathol. DOI: 10.1080/03079450802632056.

Wei, F., J. Yang, Y. Wang, H. Chen, Y. Diao, and Y. Tang, 2020: Isolation and characterization of a duck-origin goose astrovirus in China. Emerg. Microbes Infect. DOI: 10.1080/22221751.2020.1765704. 
Wen, X., D. Zhu, A. Cheng, M. Wang, S. Chen, R. Jia, M. Liu, K. Sun, X. Zhao, Q. Yang, Y. Wu, and X. Chen, 2018: Molecular epidemiology of duck hepatitis a virus types 1 and 3 in China, 2010-2015. Transbound. Emerg. Dis. DOI: 10.1111/tbed.12741.

Willcocks, M.M., T.D.K. Brown, C.R. Madeley, and M.J. Carter, 1994: The complete sequence of a human astrovirus. J. Gen. Virol. DOI: 10.1099/0022-1317-75-7-1785.

Yang, J., J. Tian, Y. Tang, and Y. Diao, 2018: Isolation and genomic characterization of gosling gout caused by a novel goose astrovirus. Transbound. Emerg. Dis. DOI: 10.1111/tbed.12928.

Zhang, Q., Y. Cao, J. Wang, G. Fu, M. Sun, L. Zhang, L. Meng, G. Cui, Y. Huang, X. Hu, and J. Su, 2018: Isolation and characterization of an astrovirus causing fatal visceral gout in domestic goslings article.Emerg. Microbes Infect. DOI: 10.1038/s41426-018-0074-5.

Zhang, R., J. Lan, H. Li, J. Chen, Y. Yang, S. Lin, Z. Xie, and S. Jiang, 2019: A novel method to rescue and culture duck Astrovirus type 1 in vitro. Virol. J. DOI: 10.1186/s12985-019-1218-5.

Figure 1 Regions of DAstV -1 infection outbreak in China. The provinces affected with DAstV -1 infection were indicated in grey.

Figure 2 Phylogenetic relationship analysis based on nucleotide sequences of ORF1a, ORF1b and ORF2 of the DAstV-SDZZ strain $\left(^{*}\right)$ and other AstVs. The trees were generated using MEGA 7.0 software and the Neighbor-joining method with 1000 bootstrap replicates. The DAstV -SDZZ isolate determined in this work is indicated by a red dot.

Figure 3 Histopathological changes in experimentally infected ducklings with DAstV-SDZZ strain. A Petechial and ecchymosis hemorrhages in liver. B Severe hemorrhage and swellings of kidneys. C Steatosis and infiltration of inflammatory cells of livers. D Necrosis and degeneration of renal epithelial cells, accompanied by infiltration of inflammatory cells and severe haemorrhage.

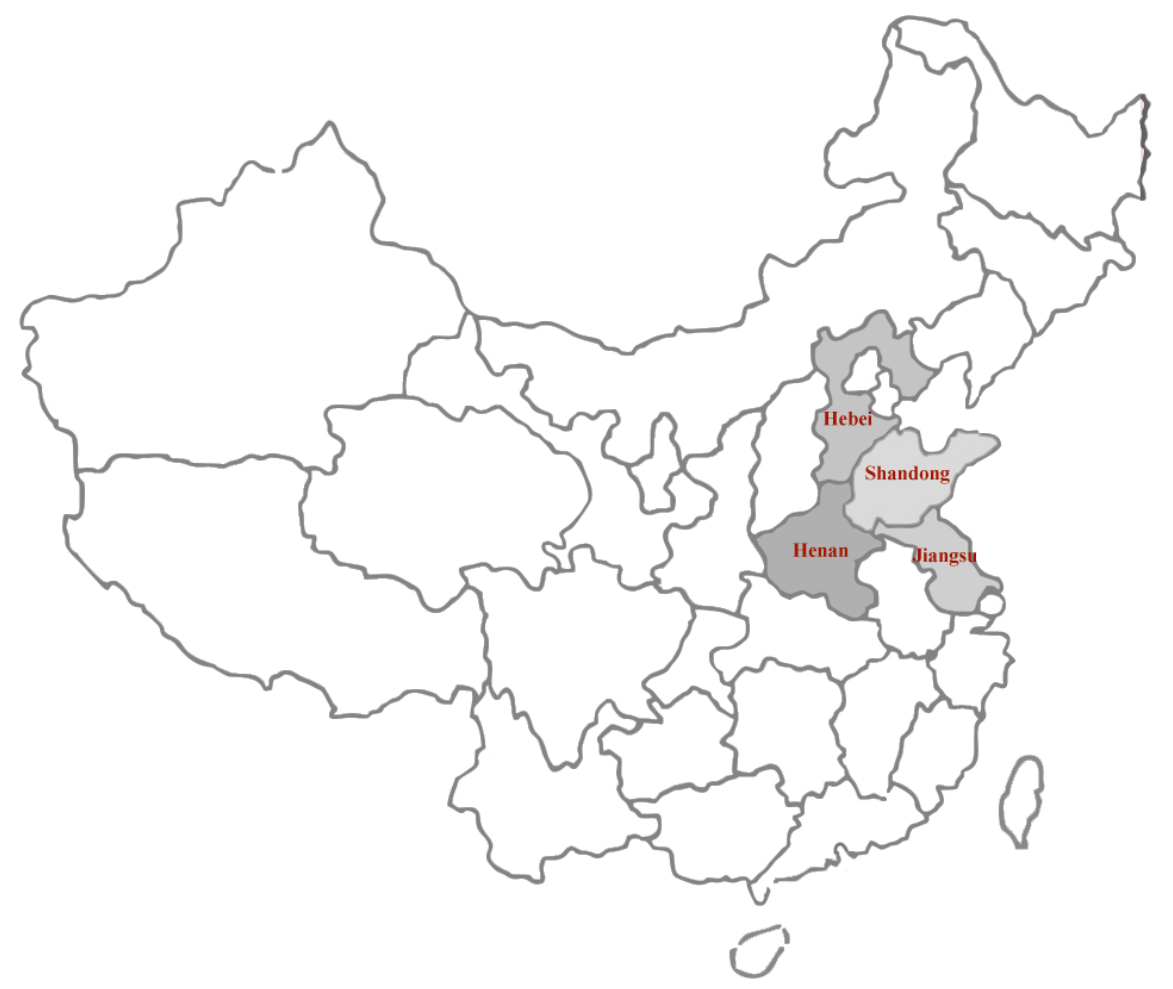


ORF 1a
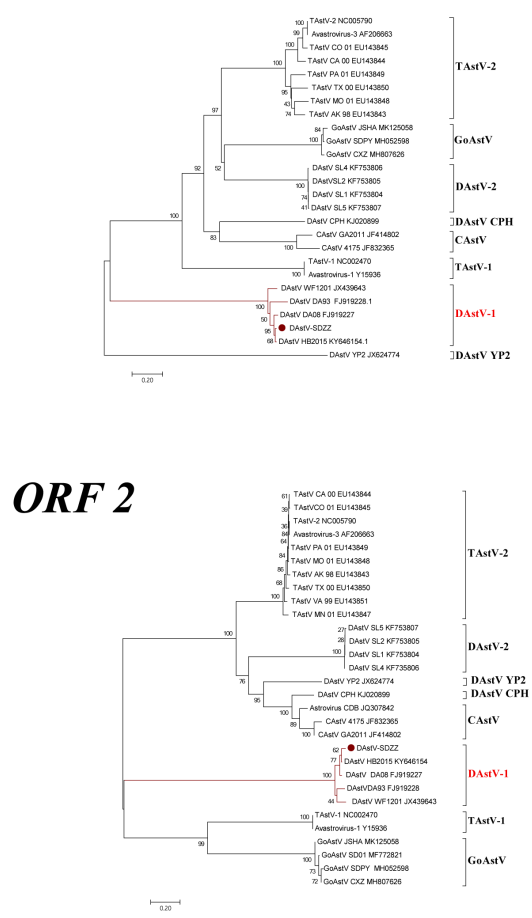

ORF $1 b$

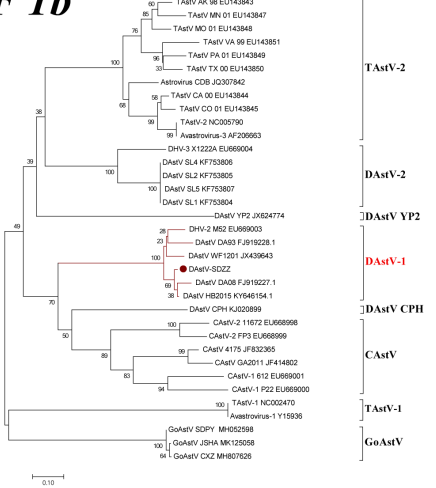

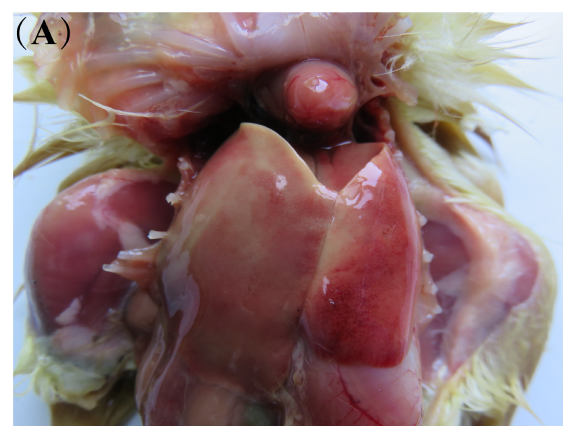
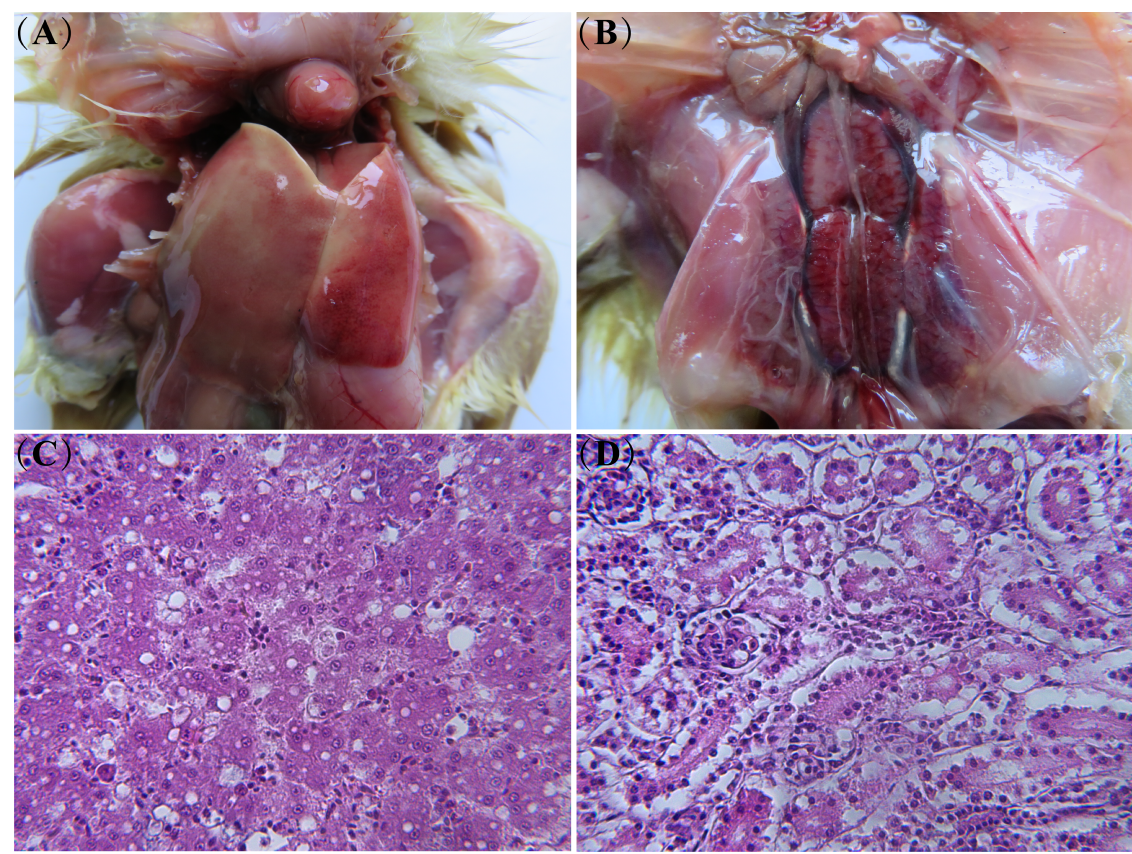

Hosted file

Table.pdf available at https://authorea.com/users/371686/articles/489923-the-isolation-andcharacterization-of-duck-astrovirus-type-1remerging-in-china 